\title{
Executive summary of the NHLBI State of the Science (SOS) Workshop: Overview and next steps in generating a national blueprint for future research on factor VIII inhibitors
}

\author{
Steven W. Pipe ${ }^{1}$ (D) | Denise E. Sabatino ${ }^{2,3}$ (D) | Diane J. Nugent ${ }^{4}$ | W. Craig Hooper ${ }^{5 *}$ | \\ J. Michael Soucie
( \\ ${ }^{1}$ Departments of Pediatrics and Pathology, University of Michigan, Ann Arbor, Michigan \\ ${ }^{2}$ Perelman School of Medicine, University of Pennsylvania, Philadelphia, Pennsylvania \\ ${ }^{3}$ The Children's Hospital of Philadelphia, Philadelphia, Pennsylvania \\ ${ }^{4}$ Children's Hospital of Orange County, University of California at Irvine, Irvine, California \\ ${ }^{5}$ Division of Blood Disorders, Centers for Disease Control and Prevention, Atlanta, Georgia \\ ${ }^{6}$ Division of Blood Diseases and Resources, National Heart Lung and Blood Institute, National Institutes of Health, Bethesda, Maryland \\ Correspondence \\ Steven W. Pipe, Departments of Pediatrics and Pathology, University of Michigan, Ann Arbor, MI. \\ Email: ummdswp@med.umich.edu \\ KEYWORDS \\ blueprint, factor VIII, haemophilia, implementation, inhibitor, research
}

\section{1 | BACKGROUND AND OBJECTIVES FOR THE NATIONAL BLUEPRINT FOR FACTOR VIII INHIBITOR RESEARCH}

As elaborated by Sabatino et al, ${ }^{1}$ five decades of advances have brought us widespread availability of safe and effective haemophilia treatment. However, inhibitors, neutralizing alloantibodies to factor VIII, still occur in $\sim 25 \%-30 \%$ of persons with severe haemophilia A. Within the US, it is estimated that there are over 1000 individuals with a factor VIII inhibitor. ${ }^{2}$ Inhibitors are associated with increased risk from bleeding and twice the rate of hospitalization for a bleeding complication, increased morbidity from joint disease and significantly increased rate of death due to bleeding-related causes compared to those without inhibitors. Over the last 30 years, we have gained insights on risk factors for inhibitor development from retrospective and parallel-cohort studies as well as meta-analyses. ${ }^{3}$ However, there is still much to be learned about the basic mechanisms underlying this immune response and few studies that have significantly impacted inhibitor prevention and eradication.

In 2017, a multi-stakeholder summit coordinated by the Centres for Disease Control's Division of Blood Disorders (DBD) identified the need for a national, coordinated strategy to develop actionable solutions

*The findings and conclusions in this report are those of the authors and do not necessarily represent the official position of the Centres for Disease Control and Prevention. to the problem of FVIII inhibitors. ${ }^{4}$ Several months later, the Medical and Scientific Advisory Council (MASAC) to the National Haemophilia Foundation formed the MASAC Inhibitor Prevention and Eradication Working Group with a mandate to engage the haemophilia community in the development of a national scientific agenda that would ensure the coordinated future conduct of the most efficient and impactful research. Discussions within this Working Group, which included the Deputy Director of the Division of Blood Diseases and Resources, National Heart, Lung, and Blood Institute (NHLBI) of the National Institutes of Health (NIH), led to the concept for The NHLBI State of the Science (SOS) Workshop entitled Factor VIII Inhibitors: Generating a National Blueprint for Future Research held on the NIH campus on May 15 and 16, 2018. The goal of this workshop was to solicit input from all constituencies within the US haemophilia community, as well as international collaborators, into the development of a coordinated and collaborative national blueprint for future basic, translational, and clinical research focused on factor VIII (FVIII) immunogenicity and FVIII inhibitor prevention and eradication. The organizational development of this workshop has been described by Sabatino et al. ${ }^{1}$

\section{2 | SUMMARY OF WORKING GROUP REPORTS}

The blueprints for future basic, translational and clinical research in FVIII immunogenicity, and in the prevention and eradication of FVIII 
inhibitors has been detailed in each of the full Working Group reports. ${ }^{5-8}$ This paper will serve to highlight and integrate several key points from each body of work.

\section{1 | Working Group 1: scientific priorities and innovative implementation strategies for FVIII inhibitor clinical trials}

This Working Group considered innovative approaches to the design and implementation of feasible clinical trials for prevention and/ or eradication of FVIII inhibitors, with special consideration of integration of novel non-factor therapeutics and gene therapy into the standard of care for FVIII inhibitor patients. ${ }^{5}$ Two proposed trials in these areas served to highlight the incorporation of innovative trial design, establishment of a functional clinical trials infrastructure, facilitation of international collaboration, support of public-private partnerships, strong patient engagement, and the training of the future workforce, as vital to a robust and well-coordinated future clinical trials enterprise in the US. In this vision, clinical trials would be integrated into and informed by a national system for data and biospecimen collection, ${ }^{6}$ and would serve as platforms for mechanistic studies of FVIII immunogenicity and the host response.,8

\section{2 | Working Group 2: scientific priorities and strategies for 21st century data and specimen collection and observational FVIII inhibitor cohort studies}

This Working Group focused on the following key areas to identify the best future state for lifespan observational cohorts. ${ }^{6}$ This group identified an existing haemophilia data/specimen collection infrastructure on which to build a nationally integrated and coordinated 21st century data and specimen collection network designed to support basic, translational and clinical research (clinical trials and observational cohort studies). This enhanced data/specimen collection and storage enterprise would leverage federally-funded resources, capitalize on informatics and biorepository experience from outside the field, serve to crosstrain haemophilia experts in data science and epidemiology, as well as support training efforts across the haemophilia community, and incorporate several progressive principles in data and biorepository science.

Data acquisition and storage would necessarily meet the NIH FAIR (Findable, Accessible, Interoperable, Reusable) guidance ${ }^{9}$ and encompass the realms of human subjects, data sharing, ethics, and both clinician- and patient (participant)- entered data collection. Within these realms, the group addressed the need for an optimized data enterprise to revisit and incorporate policies and procedures related to the design of studies and informed consent for long term use of data; single institutional review board review of studies; international sharing of anonymized data; flexible database architecture; software interfaces to facilitate interoperability between clinical and research databases; and the consistent use of standard measures and high quality patient-relevant outcome (PRO) elements and instruments for both data collection and the definition and analysis of outcomes.
Centrally regulated biospecimens acquisition and storage would encompass the capacity to store and efficiently distribute phenotypically-linked biospecimens to investigators for use in reviewed and approved scientific studies; to establish standardized processes for on-site small volume specimen collection, storage and shipment; and to coordinate efforts with NHLBI's BioLINCC, an archival datalinked biospecimen repository. ${ }^{10}$

\section{3 | Working Group 3: scientific priorities and implementation strategies for acquiring an actionable understanding of FVIII immunogenicity and the immunology of both the host immune response and tolerance}

This Working Group proposed basic and translational research on the immune response to factor VIII that would explore both the mechanisms of the initial immune response to FVIII that results in peripheral tolerance or inhibitor development, and the mechanisms by which the immune system responds to FVIII exposure within immune tolerance regimens following initial inhibitor development. ${ }^{7}$ Three scientific priorities were identified:

1. Activation signals and immune regulation that shape the response to FVIII in humans.

2. Utility of animal models and non-animal approaches (in silico, genetic, -omics, single cell, and other in vitro) to help predict inhibitor formation and identify novel therapeutics.

3. Impact of the source of FVIII, its structure, and von Willebrand factor (VWF) on immunogenicity and tolerance (including the impact of gene therapy on FVIII tolerance versus immunogenicity).

Addressing these questions would require that mechanistic studies be integrated into clinical antenatal/neonatal and lifespan cohort studies and into clinical trials through which biospecimens linked to patient phenotype and key clinical events would be collected. ${ }^{5,6,8}$ As part of their proposed implementation plan, this Working Group identified the opportunities for emerging workforce training in team science that would draw various disciplines, including but not limited to biochemistry, genetics, genomics, bioinformatics, and immunology.

\section{4 | Working Group 4: design of pregnancy/birth longitudinal cohorts that leverage omics, existing phenotypic data, and in silico modelling to study FVIII immunogenicity, as well as inhibitor development and eradication}

This Working Group was asked to consider the design of a pregnancy/birth longitudinal cohort that would leverage -omics, existing phenotypic data, and in silico modelling to elucidate mechanisms of immunogenicity and tolerance to FVIII exposure would inform the development of prediction tools for inhibitor risk, strategies to avoid modifiable risk factors, and novel interventions during the highest risk period. ${ }^{8}$ Their mandate included 
the design of data capture and mechanistic studies, based on the translational scientific priorities required to build precision (personalized) medicine-based clinical algorithms that can be applied across the lifespan.

This group recommended that a longitudinal study beginning in the antenatal period with the enrolment of known carriers for severe haemophilia A during pregnancy, and continuing with the follow-up of affected offspring through the highest risk period for inhibitor development, would offer the first insights into the factors that influence inhibitor development prior to the first exposure. Technical advances in -omics and computational biology, as well as the previously unexplored potential contributions of foetal and placental biology, would be brought to bear on the study of this unique cohort to better understand, predict, and treat haemophilia inhibitor formation and understand tolerance. Such a study could be best accomplished through novel interdisciplinary efforts, and through patient community engagement to overcome the barriers to enrolment. The infrastructure required to support such a cohort and integrate the required mechanistic studies were informed by the deliberations of Working Groups 2 and 3.6,7

\section{3 | OVERVIEW OF THE INTEGRATED BLUEPRINT FOR FACTOR VIII INHIBITOR RESEARCH IN THE US}

The overarching theme that emerged from the SOS Workshop was the idea of inhibitor investigation over the lifespan (Figure 1). New insights on inhibitor development will require holistic research investigation that encompasses the antenatal period through perinatal/neonatal transition, and early childhood through late adulthood. Research programs would ideally have the following characteristics: (a) they would study the entirety of the lifespan and include longitudinal surveillance and assessment of outcomes on a national scale; (b) they would enable subject discovery for observational studies; (c) they would facilitate subject discovery from within observational cohorts; and (d) they would ideally encompass research into the developmental origins of health and disease (DOHaD).

At any point in time, persons with haemophilia can be identified within one of three "swim lanes", or cohorts, based on their history of exposure and inhibitor status-those who are at risk for an inhibitor, those who have developed an inhibitor or those who are tolerant to FVIII (Figure 1). The broadest participation would be at the registry and biorepository level. Over the course of the lifespan, individuals would move between these swim lanes according to their FVIII exposure and the evolution of their immune response. Regardless of their prior history, individuals may enter the observational cohorts into whichever swim lane is consistent with their clinical status. For example, newly diagnosed infants may be identified de novo or from known carriers and be part of observational cohort studies to identify biomarkers for inhibitor development. Those subjects with positive biomarkers could be recruited into clinical trials for inhibitor prevention. Following clinical trial intervention, those subjects could return to their observational cohorts within their new swim lanes or subsequently be recruited into clinical trials for inhibitor eradication. The fluid movement from

Inhibitor investigation over the lifespan

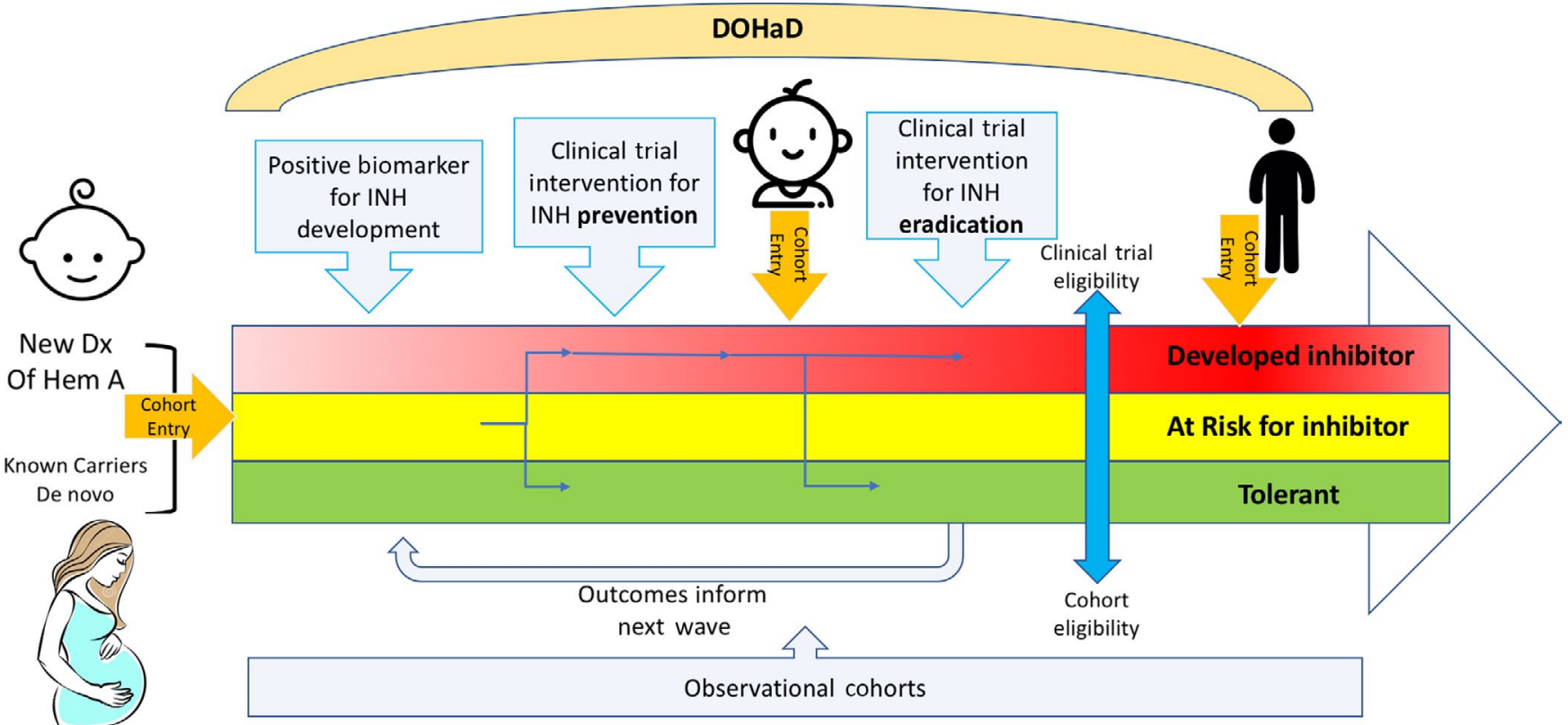

Scientific Priorities:

1. Longitudinal outcomes/surveillance

2. Observational Cohort Discovery
3. Subject Discovery for Clinical Trials

4. Developmental Origins of Health and Disease (DOHaD)

FIGURE 1 Schematic for inhibitor investigation over the lifespan 
observational cohort to clinical trial participation and back to observational cohort with the longitudinal insights from the entirety of their clinical phenotyping and personalized -omics would be particularly powerful. Outcomes from basic/translational science, observational cohorts and clinical trials would then inform subsequent observational cohorts and subsequent biomarker studies and clinical trial designs. New subjects can enter these same observational cohorts at any time over the course of their lifespan as they would be able to contribute to the scientific studies within their respective cohorts. The ability to obtain data "snapshots" of the status of subjects across all the cohorts/swim lanes would allow for reporting the change in inhibitor development and eradication over time on a national scale.

\section{4 | IMPLEMENTATION PLAN}

Each of the Working Groups provided recommendations on the priorities for implementation of this national blueprint. ${ }^{5-8}$ Next steps included organizational structure and oversight, infrastructure needs, funding strategies, as well as training opportunities and needs.

\section{1 | Steering committee}

Priority should first be given to establishing a Steering Committee focused on inhibitor prevention and eradication. This Steering Committee should include investigators and representatives from the USHTCN, government agencies, database networks, community representatives, industry and insurers. Their primary responsibility would be to provide oversight for the implementation plan, prioritize infrastructure needs, to identify possible funding sources, and to develop a process for establishing observational cohorts and reviewing proposed protocol concepts.

\section{2 | Infrastructure needs}

A Haemophilia Clinical Trials Group (HCTG) concept was proposed from Working Group $1^{5}$ which would provide the resources for clinical trial infrastructure. This would facilitate funding for personnel, data acquisition, sampling, processing and shipment; set up a single IRB for protocol submission; drive trial implementation; and assure appropriate clinical trial design and biostatistical support for trial concept development.

Working Group $2^{6}$ highlighted key points for strategic implementation that include improvements that build on existing clinical databases; establishment and optimization of automated clinician- and patient-entered data transfer; use of standard measures and PROs across studies for harmonization of clinical phenotypes and outcomes to facilitate data integration; streamlining of protocols, contracting and informed consent processes; and centralized tracking of biorepository specimens and data.

A recent collaborative research initiative, the NHLBI-funded (U54) Centres for the Investigation of Factor VIII Immunogenicity, was launched in 2018. ${ }^{11}$ These Centres will utilize cross-disciplinary science and novel technologies to define the basic mechanisms involved in the development of inhibitors to FVIII. Three centres have been awarded funding to characterize the functional repertoire and ontogeny of FVIII humoral immunity across species and to study the in vivo mechanisms of FVIII immunity and the influence of the host microbiome. Further, there will be efforts to define the structural basis for FVIII immune recognition and related immunobiology, to characterize the immunopharmacology of FVIII bioengineering and gene therapy to study the role of glycosylation in inhibitor formation, and to characterize genetic effects on FVIII glycosylation patterns and inhibitor formation. These U54 centres will provide insights from basic and translational science that will inform the architecture of the clinical research within the observational cohorts and interventional trials over the lifespan. The results from clinical research will in turn drive new directions in the basic and translational science. The novel team science and crossdisciplinary infrastructure proposed by Working Group $3^{7}$ would build on these efforts.

The novelty of the antenatal/neonatal observational cohort, as proposed by Working Group $4,{ }^{8}$ presents new challenges for our research infrastructure. They have highlighted the need for new systems to recruit carriers and enrol subjects, unique biospecimens and real-time sample acquisition that are suitable to allow for integration of -omics science and computational biology, as well as the need for cross-disciplinary scientific engagement to explore the role of maternal factors and placental biology in contributing to the FVIII immune response.

\subsection{Funding strategies}

The USHTCN receives federal funding through cooperative grant agreements from Health Resources \& Services Administration and the CDC. However, support for longitudinal cohorts over the lifespan will require considerably more resources. The NHLBI has recently announced several initiatives that could accelerate implementation of the national research blueprint beginning with a request for information to solicit input on FVIII immunogenicity and FVIII inhibitor prevention/eradication in patients with haemophilia A. ${ }^{12}$ This is an important step in helping NHLBI prioritize funding opportunities in the years ahead. NHLBI has also established the Innovative Clinical Trials Resource (ICTR) to provide infrastructure and expertise to support awardees of the NHLBI "Catalyzing Innovation in Late Phase Clinical Trial Design and Statistical Analysis Plans Initiative."13,14 This initiative will provide funding support for innovative design and statistical planning for late phase (phase II and beyond) single-site or multi-site investigator-initiated clinical trials. NHLBI has also announced their intent to publish a funding opportunity announcement for rare disease cohorts in heart, lung, blood and sleep disorders. ${ }^{15}$ This planned initiative will provide opportunities to efficiently advance rare disease research using genetics and deep phenotyping to characterize the disease and to identify disease sub-types and to use data science methods 
that integrate clinical and patient-reported outcomes (PROs) with laboratory, imaging and -omics data to understand the natural history of disease. The PhenX Toolkit Inhibitor Standard Measures Project, ${ }^{16}$ sponsored by NHLBI, has proposed standard measures for future haemophilia inhibitor research studies to be added to the NIH Toolbox ${ }^{17}$ with a similar project with broader scope within haemophilia planned. Public-private partnerships could also help accelerate implementation of the national blueprint. Industry and community foundations, including those representing patients, could help fund clinical trials, data networks and personnel across the haemophilia community to execute the studies that will ultimately influence the future standard of care for persons with haemophilia.

\section{4 | Training opportunities and needs}

There will be a need to train providers and patients about the best strategies for incorporating research with rigorous methodology into the HTC culture. To properly study the FVIII immune response as proposed by this blueprint would require a commitment to research from the first visit, at which persons with haemophilia (PWH) would be educated about how they can contribute to research throughout their lifespan through a national registry, prospective cohorts, and of clinical trials.

Training opportunities would be embedded within clinical trials. Senior investigators should invite young investigators to participate in NHLBI early phase and late phase single and multi-site clinical trials ${ }^{18-21}$ as well as to participate on core committees within the HCTG. They would also be embedded in basic and translational research programs. As an example, NHLBI U54 centres have been structured to include skill development cores that will aid in the training of interdisciplinary scientists who can then be better equipped to develop future studies on FVIII immunogenicity and tolerance. ${ }^{11}$

Additionally, attention would be given to training grants that are focused on post-doctoral cross- training in epidemiology, data science, clinical trial methodology, immunology, placental biology, -omics, computational biology and haemostasis. These training opportunities can leverage NIH National Research Service Award (NRSA) and Career Development Award funding mechanisms and Physician Scientist Training Programs focused on translational research in paediatrics, obstetrics and gynaecology and immunology, as well as the range of pilot awards currently offered by existing foundations, professional societies and industry. ${ }^{22}$

\section{5 | THE CRUCIAL IMPORTANCE OF HAEMOPHILIA COMMUNITY ENGAGEMENT}

The engagement of the entire haemophilia community is crucial to the success of the future national research enterprise. $\mathrm{PWH}$ and their families are at the core of data and sample collection within this national blueprint for inhibitor research over the lifespan. Ideally, the perspective of PWH would be sought and considered within all of the scientific objectives within this national blueprint. Through improved involvement of $\mathrm{PWH}$ and their families, clinical trials and protocols will be designed to better reflect patient requirements and conducted with greater consideration of patient circumstances. This will help determine which burdens of disease matter most and what aspects of clinical trials can be tailored for the $\mathrm{PWH} /$ subject. In addition, families will better understand the importance of clinical trials and how their participation will help improve care for haemophilia. ${ }^{23-25} \mathrm{PWH}$ have a unique perspective and will consider issues differently than regulators, manufacturers, scientists, clinicians and payers. Thus, this perspective needs to be considered throughout the process of selecting outcomes of importance and the development of PRO instruments. Feedback of research results directly to patients improves patient satisfaction with research, would ensure that PWH have a better understanding of the impact of their contribution to research, and likely increases the likelihood of future participation in research. Unique to this national blueprint for research is the need for identification of female carriers to develop the antenatal/birth cohort. This will likely require outreach and greater awareness within the community. Collaborative efforts with patient organizations such as the National Haemophilia Foundation, Haemophilia Federation of America and the World Federation of Haemophilia will be a key aspect of patient engagement, recruitment and the return of research results.

\section{6 | CONCLUSION}

The NHLBI State of the Science Workshop on FVIII Inhibitors was a multi-stakeholder, collaborative initiative that has generated a national blueprint for the future of research on FVIII immunogenicity with a goal towards the prevention and eradication of inhibitors for persons with haemophilia A. The proceedings represented in this overview have been archived at the NHLBI. ${ }^{26,27}$ We wish to thank the NHLBI administrative staff, the Working Group Chairs, all the Working Group Members, and all of the participants who contributed to this work.

\section{DISCLOSURES}

DJ Nugent is a member of the advisory board for NovoNordisk factor 13 and Bayer. All other authors have no conflicts of interest to declare.

\section{ORCID}

Steven W. Pipe (iD https://orcid.org/0000-0003-2558-2089

Denise E. Sabatino iD https://orcid.org/0000-0002-6116-5453

\section{REFERENCES}

1. Sabatino DE, Pipe SW, Nugent DJ, et al. Origins and organization of the NHLBI State of the Science (SOS) Workshop: generating a 
national blueprint for future research on factor VIII inhibitors. Haemophilia. 2019;25(4):575-580.

2. https://www.cdc.gov/ncbddd/hemophilia/inhibitors.html. Accessed November 17, 2018.

3. Garagiola I, Palla R, Peyvandi F. Risk factors for inhibitor development in severe hemophilia A. Thromb Res. 2018;168:20-27.

4. Making strides toward preventing inhibitors in bleeding disorders. https://www.cdc.gov/features/preventing-inhibitors/index.html. Accessed November 17, 2018.

5. Ragni M, George L; on behalf of the Members of Working Group 1. The national blueprint for future factor VIII inhibitor clinical trials: NHLBI State of the Science (SOS) Workshop on factor VIII inhibitors. Haemophilia. 2019;25(4):581-589.

6. Konkle BA, Recht M; on behalf of the Members of Working Group 2. The national blueprint for 21st century data and specimen collection and observational cohort studies: NHLBI State of the Science (SOS) Workshop on factor VIII inhibitors. Haemophilia. 2019;25(4):590-594.

7. Meeks SL, Herzog RW; on behalf of the Members of Working Group 3. The national blueprint for future basic and translational research to understand factor VIII immunogenicity: NHLBI State of the Science (SOS) Workshop on factor VIII inhibitors. Haemophilia. 2019;25(4):595-602.

8. Johnsen JM, Brown DL; on behalf of the Members of Working Group 4. The national blueprint for pregnancy/birth longitudinal cohorts to study factor VIII immunogenicity: NHLBI State of the Science (SOS) Workshop on factor VIII inhibitors. Haemophilia. 2019;25(4):603-609.

9. Wilkinson MD, Dumontier M, Aalbersberg IJ, et al. The FAIR Guiding Principles for scientific data management and stewardship. Sci Data. 2016;3:160018.

10. BioLINCC - Biologic Specimen and Data Repository Information Coordinating Center. https://biolincc.nhlbi.nih.gov/home/. Accessed November 17, 2018

11. https://grants.nih.gov/grants/guide/rfa-files/RFA-HL-18-014.html. Accessed November 17, 2018

12. https://grants.nih.gov/grants/guide/notice-files/NOT-HL-18-652. html. Accessed November 17, 2018.

13. https://grants.nih.gov/grants/guide/pa-files/PAR-18-768.html. Accessed November 17, 2018.

14. https://grants.nih.gov/grants/guide/rfa-files/RFA-HL-19-027.html. Accessed November 17, 2018.

15. https://grants.nih.gov/grants/guide/notice-files/NOT-HL-18-624. html. Accessed November 17, 2018.
16. https://original-phenxtoolkit.rti.org/index.php. Accessed November 17, 2018.

17. http://www.healthmeasures.net/explore-measurement-systems/ nih-toolbox. Accessed November 17, 2018.

18. https://grants.nih.gov/grants/guide/pa-files/PAR-18-683.html. Accessed November 17, 2018.

19. https://grants.nih.gov/grants/guide/pa-files/par-18-406.html. Accessed November 17, 2018.

20. https://grants.nih.gov/grants/guide/pa-files/PAR-18-407.html. Accessed November 17, 2018.

21. https://grants.nih.gov/grants/guide/pa-files/PAR-18-410.html. Accessed on November 17, 2018.

22. https://researchtraining.nih.gov. Accessed November 17, 2018.

23. Hoos A, Anderson J, Boutin M, et al. Partnering with patients in the development and lifecycle of medicines: a call for action. Ther Innov Regul Sci. 2015;49(6):929-939.

24. Leese J, Macdonald G, Kerr S, et al. 'Adding another spinning plate to an already busy life'. Benefits and risks in patient partner-researcher relationships: a qualitative study of patient partner's experiences in a Canadian health research setting. BMJ Open. 2018;8(8):e022154.

25. Day S, Jonker AH, Lau PL, et al. Recommendations for the design of small population clinical trials. Orphanet J Rare Dis. 2018;13(1):195.

26. State of the science inhibitors workshop: FVIII inhibitors: generating a national blueprint for future research (day 1). https:// videocast.nih.gov/Summary.asp?Live $=27769 \& b h c p=1$. Accessed November 17, 2018

27. State of the science inhibitors workshop: FVIII inhibitors: generating a national blueprint for future research (day 2). https:// videocast.nih.gov/summary.asp?Live $=27775 \& b h c p=1$. Accessed November 17, 2018.

How to cite this article: Pipe SW, Sabatino DE, Nugent DJ, et al. Executive summary of the NHLBI State of the Science (SOS) Workshop: Overview and next steps in generating a national blueprint for future research on factor VIII inhibitors.

Haemophilia. 2019;25:610-615. https://doi.org/10.1111/ hae.13713 